\title{
Surface Modification of Cellulosic Fabric
}

\author{
Ravikant Sharma ${ }^{1}$, Usha Sayed ${ }^{2}$
}

\begin{abstract}
The affinity of anionic dyes for cotton can be improved by adding cationic sites to the fiber. Various cationising agents have been employed on cotton and dye uptake and fastness properties are reported for two different reactive dyes and one acid dye. Satisfactory dye yields and colour fastness properties were obtained without the use of salts and without multiple rinsings which are generally employed in dyeing of cotton. Most of the conventional finishing agents used for easy-care are formaldehyde based cross-linkers which improve wrinkle recovery angle (WRA) and durable press (DP) but at the cost of strength and also releases formaldehyde which is a known carcinogen. Also most of traditional durable press finishing agents and flame retardants are either non-durable or environmentally unsafe or expensive. Ionic cross-linking can be the potential solution to these problems. In the method followed, cotton fabric was imparted anionic or cationic sites, using natural bio-polymer like chitosan and other agents. These sites are durable and covalently bonds with the cellulose. Various agents were then applied onto the modified cotton fabric to get desired easycare and/or flame-retardant and/or anti-microbial properties. There is no later release of hazardous chemicals also there is increase in tensile strength properties as compared to the untreated cotton fabric.
\end{abstract}

Keywords - 2-chloro-3-hydroxy-trimethylpropyl ammonium chloride (CHTAC) (64\% aqueous solution), Chitosan,poly(2hydroxyethylmethacrylate).

\section{INTRODUCTION}

C ELLULOSE is a macromolecule made up of anhydroglucose units united by 1,4 , oxygen bridges as shown in Figure 1. The anhydroglucose units are linked together as beta-cellobiose; therefore, anhydro-beta-cellobiose is the repeating unit of the polymer chain. The number of these repeats units that are linked together to form the cellulose polymer is referred to as the degree of polymerization and is between 1000 and 15000 .

Reactive dyes are best as they can be applied by various methods and give better fastness properties. The exhaustion of the dye onto the fiber requires large quantity of salt. The salt used is not exhausted onto the fiber and remains in the dye-bath. Also the dye exhaustion onto the fiber is only

\footnotetext{
${ }^{1}$ Research Scholar,Department of Fibers and Textile Processing Technology,Institute of Chemical Technology,Nathalal Parekh Marg,Matunga,Mumbai,Maharashtra,India

${ }^{2}$ Associate Professor, Department of Fibers and Textile Processing Technology,Institute of Chemical Technology,Nathalal Parekh Marg,Matunga,Mumbai,Maharashtra,India.
}

around $60 \%$ to $70 \%$. The left over unexhausted dye solution is discharged as effluent, thereby polluting the environment. The Cationic modified cotton require lesser soda ash for fixation, lesser water for rinsing and also time duration for dyeing is short. The cationic modified cotton can also be dyed with acid dyes. The cotton can also be anionised, by doing so it can also be dyed with the basic dyes without mordanting and better substantivity can be attained. [1].

\subsection{Chitin and Chitosan}

Chitin, poly- $(1,4)-2$ acetomido-2-deoxy;- $\beta$-D-glucose, is the second most abundant polymer [2]. Chitosan is the deacetylated form of chitin, i.e. poly- $(1,4)-2$-deoxy- $\beta$-Dglucose.Chitin and Chitosan are widely distributed in animals and fungi and are the basic polysaccharides that are the major component of the shells of crustacean such as crab, shrimp and Cray fish.

The following is a chronological order of the processes needed to produce Chitosan from crustacean shells

Crustacean Shell $\rightarrow$ Size reduction $\rightarrow$ Protein separation $(\mathrm{NaOH}) \rightarrow$ Washing $\rightarrow$ Demineralization (HCL) $\rightarrow$ Washing and Dewatering $\rightarrow$ Chitin - deacetylation $(\mathrm{NaOH}) \rightarrow$ Washing and Dewatering $\rightarrow$ Chitosan

\subsection{Properties of Chitosan}

$>$ Solution properties of chitosan in free Amine $\left(-\mathrm{NH}_{2}\right)$ form soluble in acidic solution.

$>$ Insoluble at $\mathrm{pH}$ 's $>6.5$ Insoluble in $\mathrm{H}_{2} \mathrm{SO}_{4}$

$>$ Limited solubility in $\mathrm{H}_{3} \mathrm{PO}_{4}$

$>$ Insoluble in many organic solvents

$>$ Soluble at $\mathrm{pH}<6.5$

$>$ Form viscous solutions

$>$ Solution shear thinning, forms gels with polyanions

$>$ Will remain soluble in some alcohol-water mixture.

\subsection{Chemical properties of Chitosan:}

Chitosan is a linear polyamine (poly-o-glucosamine) with reactive hydroxyl and amine group. Biocompatility, Cicatrizing,Anti-cholesterolemic agent,Chelation agent, Biodegradable, Strengthening the immunity, Antimicrobial activity, Deodrant properties of Chitosan, Water Treatment and Pollution Control [3]

\subsection{Application of Chitosan in Textile Wet Processing}

Chitin and chitosan have higher affinity for dyes and metals and certain surfactants, which contribute to water pollution. Using the shellfish waste thus has two fold advantages:-

A) First to find a viable method to purify dye waste.

B) To use natural resources, this could otherwise have been wasted. 
C) After use for color removal the spent sorbent further finds uses as a raw material for papermaking.

The use of chitosan as a combined thickener and binder in pigment printing: Printing paste made from chitosan, acetic acid and pigments at appropriate viscosity give stable pastes and satisfactory results on polyester and P/C blends. Chitosan can also used in the dye bath, because due to the unimolecular structure it has an extremely high affinity for many classes of dyes including disperse, direct, reactive, acid, vat, sulphur and basic etc. At lower $\mathrm{pH}$ chitosan free amines is protonated causing attracting anionic dyes [4].

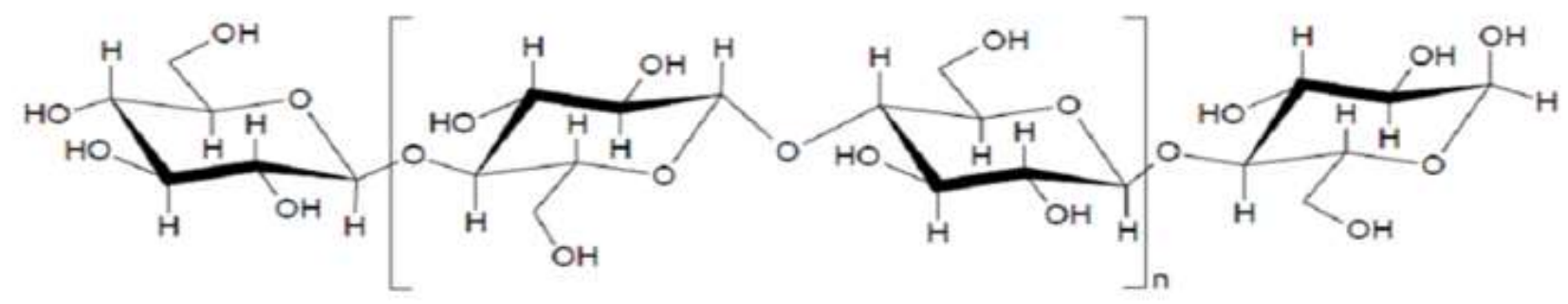

Fig. 1: Chemical structure of cellulose

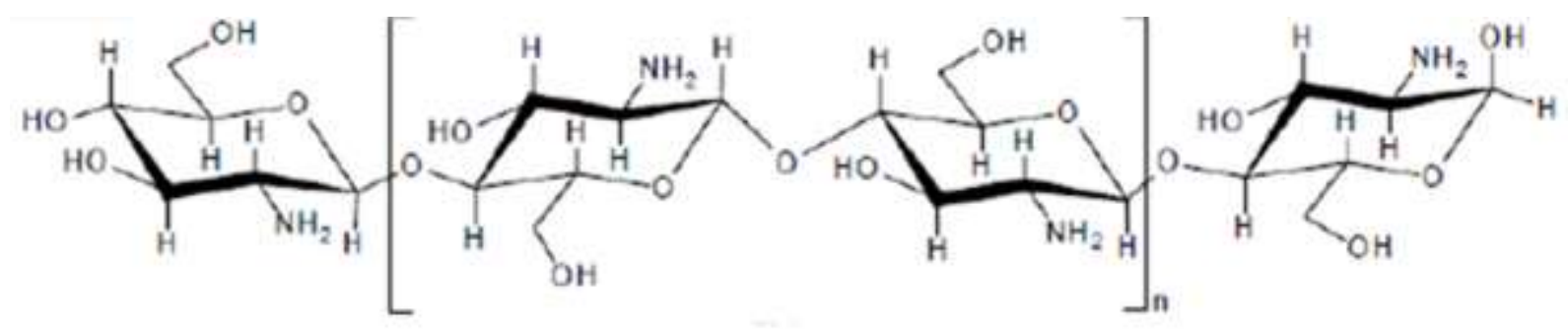

Fig. 2: Chitosan

\section{SCOPE OF THE WORK}

The objective of this research was to modify the cotton substrate ionically, which can impart durable finish to the cotton substrate. Study is also done to check the behavior of the ionically modified cotton towards various dyes. It has been found that catatonically modified cotton can be easily dyed with the Reactive dyes without use of salt and less alkali for fixation. Cationised cotton fabric can also be dyed with acid dyes with very good exhaustion but with lesser fastness properties.

\section{MATERIALS AND METHODS}

\subsection{Material Used}

Fabric: $100 \%$ Bleached Cotton fabric

Cationising Agent: 2-chloro-3-hydroxy-trimethylpropyl ammonium chloride (CHTAC) (64\% aqueous solution) was sourced from Dow Chemical Company.

Chitosan: Microcrystalline Chitosan Powder (100 Mesh Size) was sourced from Mahatani Chitosan Pvt. Ltd.

\subsection{Anionic Modification of Cotton}

The initial procedure for this research was the optimization of the anionic modification process. This procedure involves three major steps. First, the fabric is treated with $\mathrm{NaOH}$.
Second, the fabric is treated with the chloroacetate, washed, treated with acetic acid, and washed again. Finally, the partially carboxymethylated fabric is treated with different synthesized polycations namely Cationised Chitosan, Fabric is treated with $\mathrm{NaOH}$ using a pad-dry method with prior soaking in the solution. The fabric is placed in a solution of $20 \%$ by weight aqueous $\mathrm{NaOH}$. The fabric is soaked for 10 minutes in this solution at room temperature. It is then padded to approximately $100 \%$ WPU. The samples are dried at $50^{\circ} \mathrm{C}[5]$.

The fabric is then treated with Sodium chloroacetate. The fabric is placed in a different molar aqueous solution of Sodium chloroacetate. The fabric is left in the solution for 5 minutes at room temperature. It is padded to approximately $100 \%$ WPU. The fabric is placed in a polyethylene bag and the air is removed from the bag. It is held in the sealed bag at $70^{\circ} \mathrm{C}$ for 60 minutes. After 60 minutes, the fabric is rinsed in warm water and then with cold water. It is treated with a $2 \mathrm{gpl}$ acetic acid solution. Finally, fabric is squeezed and dried at room temperature for 24 hours [5].

The treated fabric is analysed for carboxymethyl group per 100 gram of fabric. The best recipe is selected for the further treatments. 
<smiles>[N]OC(=O)CCl</smiles>

Chaloroacetic Acid(Sodium Salt)

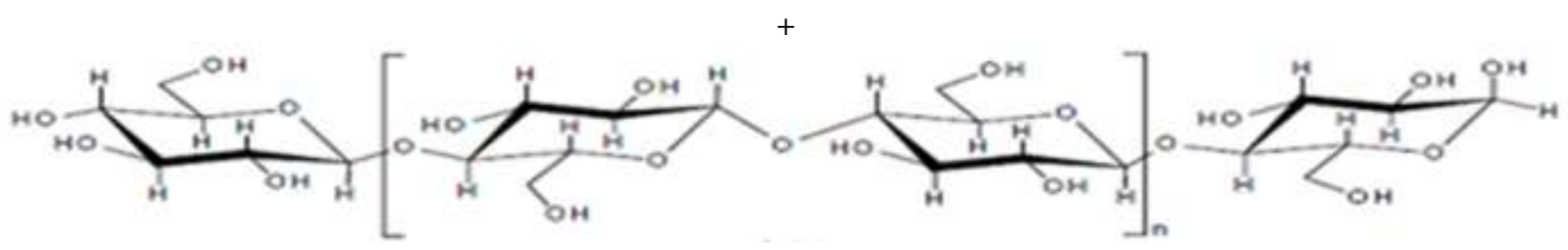

Cellulose

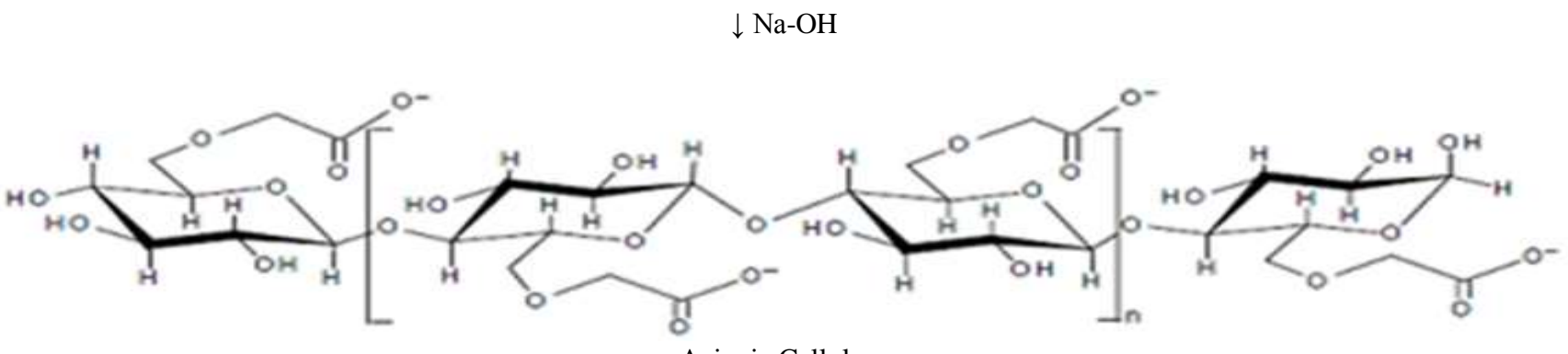

Anionic Cellulose

Fig. 3: Anionic Modification of Cotton Using Sodium Chloroacetic Acid

\subsection{Cationic Modification of Cotton:}

Cotton fabric is modified with cationising agents namely 2chloro-3-hydroxy-trimethylpropyl ammonium chloride (CHTAC). These cationised fabrics are dyed with reactive dye and the one with highest dye uptake is considered to have maximum cationisation and hence selected for the further ionic cross linking treatment using 2-chloro-3-hydroxy- trimethylpropyl ammonium chloride (CHTAC).

The cationization of cellulose using CHTAC basicly takes place in two stages. Sodium hydroxide $(\mathrm{NaOH})$ is the base catalyst. The cationic character of cellulose is independent from $\mathrm{pH}$. In the first stage the epoxide form of CHTAC formed in the presence of $\mathrm{NaOH}$. In the second stage this epoxide reacts with a hydroxyl group in the Cellulose.<smiles>C[N+](C)(C)C[C@@H](O)CCl</smiles>

3-Chloro-2-hydroxypropyl Trimethylammonium Chloride (CHTAC)

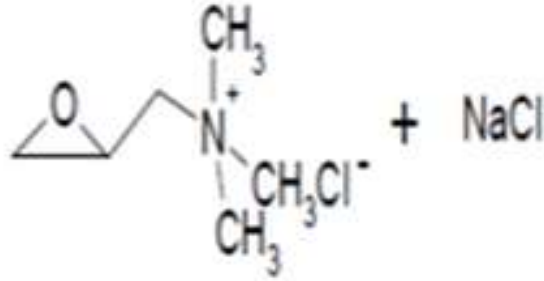

2, 3-epoxypropyl trimethyl ammonium chloride

Fig. 4: Conversion of CHTAC to 2, 3-epoxypropyletrimethylammoniumchloride in the presence of $\mathrm{NaOH}$ 

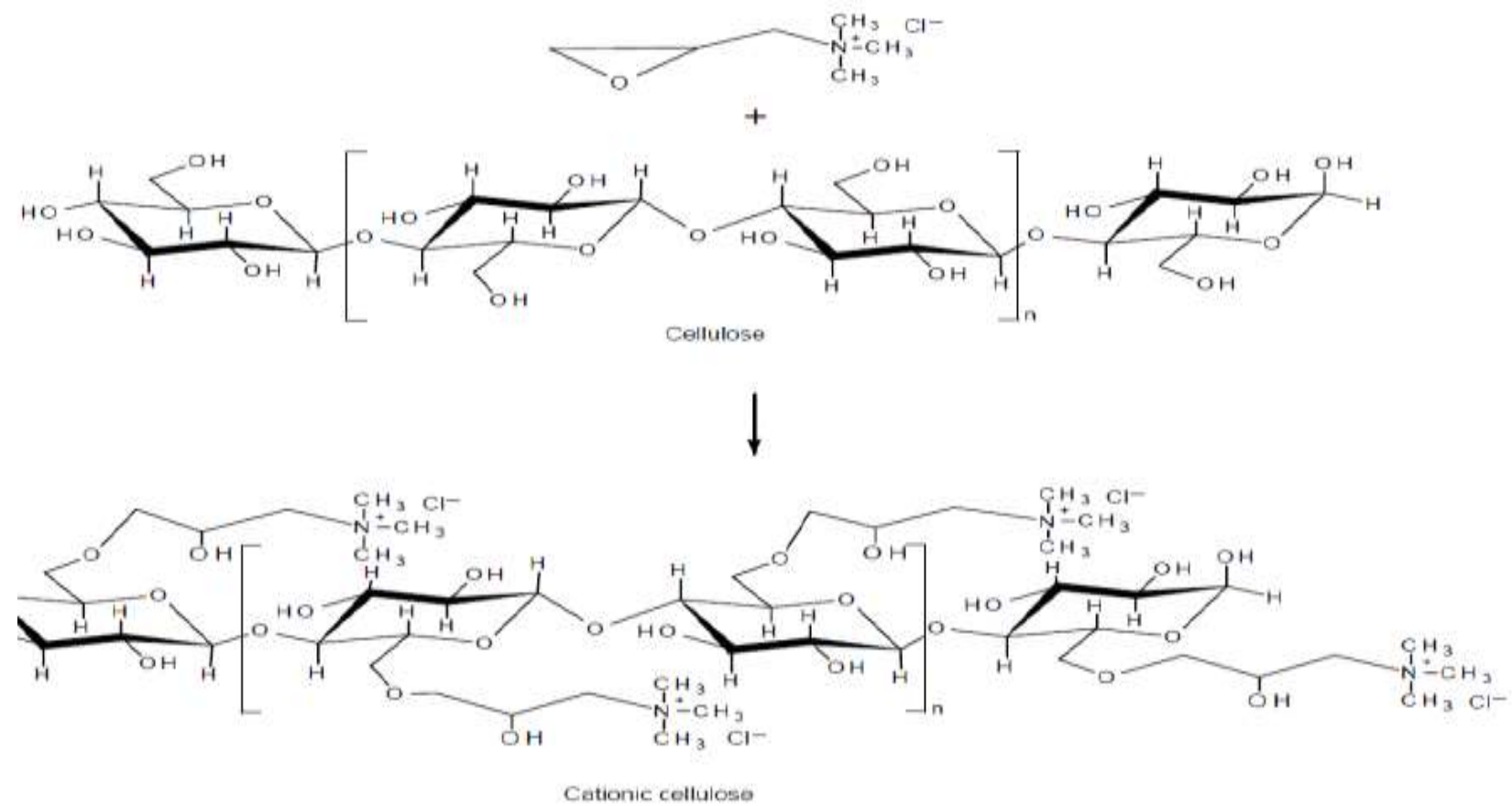

Fig. 5: Cationic modification of cotton using CHTAC

Only exhaustion method of application is explored. Cotton is treated with different concentration of alkali and CHTAC, and in different sequence. Treated fabric is then washed with warm water, cold water, neutralized with $2 \mathrm{gpl}$ acetic acid, rinsed with cold water and finally dried at room temperature.

\section{RESULTS AND DISCUSSION}

\subsection{Anionic Modification of Cotton}

Analysis for Carboxymethylation: The carboxylic acid group content of the partially carboxymethylated cellulosic fabrics were determined. Cotton fabrics were cut into small pieces, $100 \mathrm{~mL}$ of $0.1 \mathrm{~N}$ aqueous $\mathrm{HCl}$ solution prepared and fabric samples were steeped in it for 12 hours. The samples were then filtered off and washed several times with distilled water until free from $\mathrm{HCl}$ and having a $\mathrm{pH}$ of 7 . Silver nitrate drop test was performed and it showed no presence of chloride. The samples were dried at 1050C for 3 hours. Accurate weight of samples (exactly $0.25 \mathrm{~g}$ each) was soaked in $25 \mathrm{~mL}$ of $0.05 \mathrm{~N}$ aqueous $\mathrm{NaOH}$ solutions at room temperature for 8 hours. First, a blank solution (solution without any sample) was titrated with $0.05 \mathrm{~N}$ aqueous $\mathrm{HCl}$ solution. Phenolphthalein $\mathrm{pH}$ indicator was used. The volume of $\mathrm{HCl}$ solution $(\mathrm{mL})$ spent was recorded for the blank. Then, each of the solutions with different carboxymethylated samples was titrated in the same way as the blank. The carboxyl contents of samples were calculated as follows: [5] mmols carboxymethyl content per 100 grams $=100 \mathrm{X}$

(Vblank - Vsample) $\mathrm{HCl}$ X NHCl / 0.25

Where, $\mathrm{V}$ blank = the volume of $\mathrm{HCl}$ used for titration of blank solution,

$\mathrm{V}$ sample $=$ the volume of $\mathrm{HCl}$ used for titration of sample solution,

$\mathrm{N} \mathrm{HCl}=$ the normality of $\mathrm{HCl}$ titrant.

As determined by titration Table shows the summary of the titration process.

\begin{tabular}{|c|c|c|c|c|c|}
\multicolumn{7}{|c|}{ TABLE 1: Results for Carboxymethylation of Cellulosic Fabrics (V blank $=23.7 \mathrm{ml})$} \\
\hline S.No. & $\begin{array}{c}\text { CAA } \\
\text { Concentration(M) }\end{array}$ & Treatment & Weight of Sample (gm) & V sample & $\begin{array}{c}\text { Carboxyl Content } \\
\mathrm{mmol} / 100 \mathrm{gm}\end{array}$ \\
\hline 1 & 0 & None & 0.25 & 23.5 & 4 \\
\hline 2 & 0.5 & Carboxymethylation & 0.25 & 22.3 & 28 \\
\hline 3 & 1 & Carboxymethylation & 0.25 & 19.4 & 86 \\
\hline 4 & 1.5 & Carboxymethylation & 0.25 & 18.2 & 110 \\
\hline 5 & 2 & Carboxymethylation & 0.25 & 17.7 & 120 \\
\hline 6 & 2.5 & Carboxymethylation & 0.25 & & 16.8 \\
\hline 7 & 3 & Carboxymethylation & 0.25 & & 138 \\
\hline
\end{tabular}


TABLE 2: Performance properties of Cellulosic Fabrics treated with cationizing agent provided by Atul Company

\begin{tabular}{|c|c|c|c|c|c|c|c|}
\hline $\begin{array}{c}\text { Product By Atul Co. } \\
\text { Conc.(gpl) }\end{array}$ & K/S & L $^{*}$ & $\mathrm{a}^{*}$ & $\mathrm{~b}^{*}$ & W.F & BL(cm) & T.S(kg) \\
\hline Std Dyed Sample & 6.06 & 52.78 & 46.96 & 16.73 & $4-5$ & 1.7 & 14.53 \\
\hline 50 & 12.06 & 55.47 & 52.25 & 22.64 & 4 & 2.5 & 12.12 \\
\hline 100 & 18.88 & 56.80 & 59.34 & 22.51 & 4 & 2.8 & 14.70 \\
\hline
\end{tabular}

TABLE 3: Performance properties of printed cotton fabric using cationizing product provided by Atul Company (effect of concentration) Dye Used: Brilliant Carsino MO Acid Dye Shade $1 \%$

\begin{tabular}{|c|c|c|c|c|c|c|c|}
\hline $\begin{array}{c}\text { Product By Atul Co. } \\
\text { Conc.(gms) }\end{array}$ & K/S & L & $\mathrm{a}^{*}$ & $\mathrm{~b}^{*}$ & W.F & BL(cm) & T.S(kg) \\
\hline 5 & 12.72 & 42.30 & 56.72 & 34.59 & 4 & 2.7 & 10.24 \\
\hline 10 & 15.91 & 42.83 & 57.79 & 34.59 & 4 & 2.8 & 13.80 \\
\hline
\end{tabular}

TABLE 4: Performance properties of printed cotton fabric using 10 gms Atul company product (effect of Temperature) Dye Used: Brilliant Carsino MO, Acid Dye Shade 1\%

\begin{tabular}{|c|c|c|c|c|c|c|c|}
\hline Temperature C & K/S & L & $\mathrm{a}^{*}$ & $\mathrm{~b}^{*}$ & W.F & BL(cm) & T.S(kg) \\
\hline 100 & 14.52 & 42.8 & 57.65 & 34.59 & 4 & 3.4 & 13.80 \\
\hline 160 & 15.91 & 41.3 & 54.70 & 32.95 & 4 & 2.8 & 11.76 \\
\hline
\end{tabular}

\section{DISCUSSION}

At the outset, cellulose fabric was treated with cationic agent with the method as given above \& dyed with Brilliant Carsino MO Acid Dye on cotton fabric by using cationic agent as seen on Table 2.There was a larger dye uptake with higher concentration of cationising agent. On printing with same cationising agent at 2 concentrations the depth of the colour increased as seen in Table 3 . The effect of temperature on $\mathrm{K} / \mathrm{S}$ values on printing on cotton fabric is as seen Table 4, increasing in temperature increase the depth of colour.

\section{Application of Chitosan}

Chitin was deacetylated to chitosan treating with $30 \%$ Sodium hydroxide at $100 \mathrm{deg}$. C for a prolonged period till the precipitate of chitosan are separated. The precipitate was further purified to get $80 \%$ purity. This was used for the treatment of cotton. A stock solution of $1 \%$ chitosan was prepared by dissolving chitosan in $1 \%$ acetic acid and filtering it to remove any insoluble. The fabric samples were treated with this solution at liquor ratio of $30: 1$ at $50^{\circ} \mathrm{C}$ for 60 minutes in rotadyer. Then the samples were squeezed and dried at room temperature.To facilitate the even application of chitosan on the fabric, it is applied along with a non ionic surfactant Auxipon NP (0.1 gram per liter concentration).

\section{Results}

TABLE 5: Performance properties of cotton pretreated with $1 \%$ acetic acid at various chitosan concentrations (dyed sample) Dye Used: Brilliant Carsino MO Acid dye

\begin{tabular}{|c|c|c|c|c|c|c|c|}
\hline $\begin{array}{c}\text { Chitosan } \\
\text { Concentration(gms) }\end{array}$ & K/S & $\mathrm{L}^{*}$ & $\mathrm{a}^{*}$ & $\mathrm{~b}^{*}$ & W.F & BL(cm) & T.S(kg) \\
\hline Std Dyed sample & 6.06 & 52.78 & 46.95 & 16.73 & 3 & 1.7 & 14.53 \\
\hline 0.3 & 11.02 & 57.4 & 51.7 & 21.3 & $3-4$ & 3.7 & 7.08 \\
\hline
\end{tabular}

TABLE 6: Performance properties of printed cotton fabric using starch thickener system (effect of chitosan concentration) Dye Used: Auramine O Brilliant Yellow Basic Dye Shade 1\%

\begin{tabular}{|c|c|c|c|c|c|c|c|}
\hline $\begin{array}{c}\text { Chitosan } \\
\text { Conc(gms) }\end{array}$ & K/S & L & a* & b* & W.F & BL(cm) & T.S(kg) \\
\hline Std printed sample & 12.2 & 80.6 & -6.37 & 82.11 & 3 & 1.4 \\
\hline 0.15 & 2.12 & 63.72 & -6.34 & 58.8 & $2-3$ & 3.20 & 7.82 \\
\hline 0.30 & 2.57 & 68.60 & -9.4 & 65.31 & $2-3$ & 3.70 & 9.34 \\
\hline 0.60 & 3.31 & 74.09 & -6.7 & 74.53 & $2-3$ & 4.20 & 21.08 \\
\hline
\end{tabular}


TABLE 7: Performance properties of printed cotton fabric using starch thickener system (effect of Hema concentration) Synthesized by Kamini Sharma Dye Used:

\begin{tabular}{|c|c|c|c|c|c|c|c|}
\hline $\begin{array}{c}\text { Auramine O Brilliant Yellow Basic Dye Shade } 1 \% \\
\text { HEMA } \\
\text { Concentration(gms) }\end{array}$ & K/S & L & $\mathrm{a}^{*}$ & $\mathrm{~b}^{*}$ & W.F & BL(cm) & T.S(kg) \\
\hline 0.5 & 8.24 & 78.41 & -7.3 & 79.5 & 3 & 2.0 & 7.95 \\
\hline 1.0 & 11.84 & 80.39 & -6.89 & 82.49 & 3 & 2.3 & 10.35 \\
\hline 2.0 & 16.59 & 83.82 & -5.47 & 88.99 & $3-4$ & 3.9 & 16.93 \\
\hline
\end{tabular}

TABLE 8: Performance properties of printed cotton fabric using 2.0 gram HEMA (effect of Temperature) Dye Used: Auramine O Brilliant Yellow Basic Dye Shade $1 \%$

\begin{tabular}{|c|c|c|c|c|c|c|c|}
\hline Temperature C & K/S & L & $\mathrm{a}^{*}$ & $\mathrm{~b}^{*}$ & W.F & BL(cm) & T.S(kg) \\
\hline 100 & 16.59 & 83.82 & -5.47 & 88.99 & $3-4$ & 3.9 & 16.93 \\
\hline 160 & 8.62 & 80.14 & -6.3 & 82.3 & $3-4$ & 3.2 & 19.44 \\
\hline
\end{tabular}

\section{DISCUSSION}

In next set of experiment chitosan is used to treat cotton as given and the results are seen in Table 5 .Chitosan gives higher dye uptake than all other agents used,although much larger in the printed sample as seen table 6. Basic dye also gives higher dye uptake on cotton fabric in the presence of chitosan as seen in Table 6,7.On printing the cotton fabric by using POLY(2-HYDROXYETHYL METHACRYLATE) [HEMA] and starch thickener for printing on cotton fabric with using dye result seen in Table 7 . It can be seen from the experiments value the chitosan has played a better role on increasing dye uptake as well as the colour value imparted to the printed fabric. Thus slightly modification of the cellulose polymer either by cationising or by treatment with chitosan can lead to environmentally friendly process decreasing the amount of dye in the waste water.

\section{CONCLUSION}

Carboxymethylation or cationisation and ionic crosslinking significantly increase the intermolecular forces between cellulose chains. These increased forces result in increased tear strength, tensile strength, and elongation. While these ionic bonds are stronger than hydrogen bonding, they can still be easily broken by common forces such as in home laundering. When these bonds reform they will maintain their new configuration just like hydrogen bonds. As a result, fabric may actually have lower DP performance and increased wrinkling over untreated fabrics. The fabric used for this work showed significant increases in physical properties after treatment by carboxymethylation or cationisation and ionic crosslinking. Every carboxymethylation or cationisation and ionic crosslinking procedure evaluated produced tensile strength, elongation, and tear strength properties which were equal to or better than the untreated fabric. In the best cases, tensile strength was increased by $25 \%$. Dry WRA can be increased or decreased through carboxymethylation or cationisation and ionic crosslinking. Under different conditions the fabric's dry WRA was increased as much at $19 \%$ or decreased as much as $47 \%$. Wet WRA is improved significantly with this treatment. DP rating is generally decreased with this treatment.
The cotton fabric was pretreated with chitosan at different concentrations in acidic $\mathrm{pH}$ using $1 \%$ acetic acid by the exhaust methods. The following observations were made on dyeing and printed with acid, reactive and basic dyes.

$>$ Percentage exhaustion of the dye bath

$>$ Percentage change in the K/S value

$>$ Wash fastness

$>$ Bending Length

$>$ Tensile Strength

- It was seen an increase in the concentration of the chitosan during pretreatment lead to an increase in percentage change in $\mathrm{K} / \mathrm{S}$, but at a concentration of chitosan of $1 \%$ or above ununiform adsorption of chitosan on fabric takes place indicated by unlevel dyeing.

- The high exhaustive group of reactive dyes showed higher dye uptake since it is bi-functional in nature and when chitosan was present on cotton substrate there is further increase in dye uptake due to additional sites created where the dye can affix itself.

\section{SCOPE FOR THE FUTURE WORK}

While dyeing with anionic dyes on cationic cotton there is reduction in dyeing time, also soda ash requirement is reduced. Acid dyes can also be dyed. Work has to be done to improve fastness properties. Optimization of dyeing time, auxiliaries required temp of dyeing etc is required. Similar treatments can also be tried for printing on modified cotton with Acid Dyes, Basic and Reactive dye, direct dye, Pigment various dyes.

\section{REFERENCES}

[1] Bilgen. "Wrinkle Recovery for Cellulosic Fabric by Means of Ionic Cross linking." s.l. : Thesis - North Carolina State University, 2005

[2] Majeti N.V. Ravi Kumar "A review of chitin and chitosan applications" Reactive \& Functional Polymers 46 pp 12000 http://dx.doi.org/10.1016/S1381-5148(00)00038-9

[3] L. Hench Larry, "Biomaterials: a forecast for the future, Chitin and chitosan: Chemistry,properties and applications" Journal of scientific \& Industrial Research Vol 63,pp 21-29, January 2004

[4] http://textileinformation.blogspot.in/2007/11/application-of-chitosan-intextile-wet.html

[5] Zhengia Wang, Peter J. Hauser, and Orlando J. Rojas, "Study on Charge Distribution of Carboxymethylated Cotton Fabric by Streaming Potential/Current Measurements", AATCC Journal of Research vol 2 pp14-15,April 2015 
[6] Chauhan B.S."Art of Dyeing"191.

[7] OktemT.,"'Coloration Technology",119,241 (2003) http://dx.doi.org/10.1111/j.1478-4408.2003.tb00179.x

[8] Kousaku O,“Textile Research Journal”, 72(2),120 (2002). http://dx.doi.org/10.1177/004051750207200205

[9] Denning R.J.,"Textile Research Journal",67,413 (1994) http://dx.doi.org/10.1177/004051759406400707

[10] Racz, I.; Borsa, J.: "Carboxymethyl cotton fabric for pesticide protective work clothing," Text. Res. J. 68,1,9,1996

[11] Seddon R., "American Dyestuff Reporter" 74,13 (1985)

[12] Kim Y.H.,Choli H.M and YoonJ.H.,"Textile Research Journal",68(6),428 (1998).

[13] Mehra R.H. and Mehra A.R.,"Colourage", 1, 59(1994). 